\title{
Teratogenic effects of two known teratogens (Nicotine and Cadmium) and prevention of such effects by addition of antioxidants in chick embryos: An evaluation of two culture systems (Micromass and in ovo culture)
}

\author{
Samreen Memon ${ }^{1}$, Margaret Pratten ${ }^{2}$ \\ IAssistant Professor, Department of Anatomy Liaquat University of Medical and Health sciences Jamshoro, \\ Pakistan \\ 2 Associate professor Department of Anatomy, School of Biomedical Sciences, University of Nottingham, UK
}

\begin{abstract}
This study aimed to evaluate effectiveness of the chick cardiomyocyte micromass (MM) system for prediction of teratogenecity of common environmental factors and prevention of such effects by addition of multivitamins. White Leghorn 5-day-old embryo hearts were dissected and disassociate to produce a cardiomyocyte cell suspension in Dulbecco's Modified Eagle's Medium. The cultures were incubated at 370C in $5 \% \mathrm{CO} 2$ in air. Microscopic observations regarding cell beating and contractility were made at 24, 48 and 144 hours post culture. Cellular viability/metabolic activity was assessed by using the resazurin reduction assay and cell total protein content was assessed by the kenacid blue assay. It was observed that cadmium chloride induced toxicity was not reversed by addition of folic acid and vitamin $C$, however the nicotine induced teratogenic effects were reversed by addition of folic acid and vitamin $C$. The results demonstrate the potential of the chick cardiomyocyte MM culture assay to identify teratogens/embryotoxins that alter morphology and function, which may result in either teratogenic outcome or causing cytotoxicity and also protection brought about by addition of multivitamins. This could form part of a screen for developmental toxicity related to cardiac function. The effects of nicotine were compared to in ovo culture also showed protective effects of multivitamins in nicotine induced teratogenecity.
\end{abstract}

Key Words: Chick cardiomyocyte, Micromass culture, environmental teratogens, multivitamins.

\section{Introduction}

The Micromass system implicates the culture of primary cells, isolated either from different organs of the developing embryos, seeded at high density [1]. The essential rule of this assay is to detect the potential of teratogens to disrupt normal differentiation of primary embryonic cells cultured in vitro. Different organs from several species such as rat, mouse [2-4]; and chick [5-11] are used animals for MM culture. The principle of chick MM assay is the use and culture of primary cells obtained from midbrain, limbs or heart of chick embryos. Once the cells are cultured in high density they can then be exposed to the test chemicals in replicates and observed for cytotoxic effects by the use of relevant endpoints, i.e. cellular differentiation, and cytotoxicity assays [12].

Cadmium chloride is a non essential heavy metal with no known biological role in humans. Environmental sources of cadmium include combustion of fossil fuel, certain agriculture fertilizers, mining residues etc [13]. It is also found in tobacco which is a significant source. Cadmium is a well known developmental toxin. Cadmium exposure gives rise to many developmental defects in chick including limb and anterior body wall defects $[14,15]$. Environmental exposure to cadmium also produces developmental defects in other species including rats [16], mouse [17], frog [18] and zebra fish [19, 20].

Maternal smoking is considered to be one of the leading causes of congenital malformations. Active and passive smoking is an important risk factor for the developing fetus in every trimester of pregnancy [21]. Smoking during the periconceptional period affects the development of major organs of the fetus including, the central nervous system, cardiovascular system and skeletal system. Moreover, children of mothers who smoke during pregnancy have an increased risk of developing behavioural problems, attention deficits, hyperactivity and learning disabilities and nicotine dependence later in life [22]. Cigarette smoke introduces at least two thousand toxins into the blood stream, among all toxins, nicotine is considered to be the major teratogenic constituent. Nicotine exerts its effects by acting on nicotinic acetylcholine receptors. These receptors in turn increase ion influx including $\mathrm{Ca} 2+$. Increased concentrations of calcium ions, disturbs intracellular signalling and cell function. As a result of disruption of cellular function, there is increased production of reactive oxygen species (ROS), leading to oxidative stress [23]. As a consequence, excessive free radicals exert their toxic effects on many cellular organelles in different tissues and cause deleterious injury. In one study conducted on 
rat brain, Gumustekin, $\mathrm{K}$. and his colleagues found a protective effect of vitamin $\mathrm{E}$ on nicotine induced damage [24]. Similarly, protective effects of vitamin C, another antioxidant, were also observed on nicotine induced oxidative stress [25]. This study is aimed at evaluation of chick heart micromass culture by detecting toxic effects of cadmium chloride and nicotine on chick cardiomyocytes cultured in vitro and to investigate any possible protective effects of antioxidants vitamin $\mathrm{C}$ and folic acid on cadmium chloride and nicotine induced toxicity. Also results of micromass culture with nicotine were compared with in ovo culture.

\section{Chemical and solutions}

\section{Materials and Methods}

Cadmium chloride, Nicotine, Folic acid, Vitamin C (ascorbic acid), Brilliant blue (Kenacid Blue, KB), Hank's balanced salt solution (HBSS), Trypsin-EDTA, Horse serum, Penicillin/streptomycin, Resazurin, Resorufin were purchased from Sigma-Aldrich (Poole, UK). Dulbecco's Modified Eagles Medium (DMEM) and L-glutamine was purchased from Cambrex Bio Sciences Wokingham, UK, Ltd. Fetal calf serum was purchased from Autogen Bioclear (Wiltshire, UK). The test chemicals were added within 30 minutes of being prepared, and applied 24 hours after the cells were cultured.

\section{Micromass Culture Preparation}

White leghorn chicken eggs purchased from Henry Stewart Co., Louth, UK) were incubated on an automatic egg rotator at $37^{\circ} \mathrm{C}$ and $100 \%$ humidity for 5 days. The day eggs were kept in incubator was designated as day 0 . The embryos were removed from the vascular network with bent forceps, and the hearts were dissected out and collected in $5 \mathrm{ml}$ of sterile $50 \%(\mathrm{v} / \mathrm{v})$ horse serum in HBSS on ice. Once collected the hearts from all the embryos were dissociated with $4 \mathrm{ml} 1 \%$ trypsin/EDTA at $37^{\circ} \mathrm{C}$ for 20 minutes agitated at every 5 minutes. The culture medium (Dulbecco's modified eagles medium (DMEM) supplemented with 10\% fetal calf serum, $200 \mathrm{mM} \mathrm{L}$-glutamine and $50 \mathrm{U} / \mathrm{ml}$ penicillin $/ 50 \mathrm{\mu g} / \mathrm{ml}$ streptomycin) was added to neutralise the activity of the trypsin, and centrifuged at RT at $1500 \mathrm{rpm}$ for 5 minutes. The pellet was resuspended in $1 \mathrm{ml}$ culture medium and $20 \mu \mathrm{l}$ of the cell suspension $(3 \times 106 \mathrm{cell} / \mathrm{s} / \mathrm{ml})$ was plated into each well of 24-well tissue culture plate. The cells were allowed to attach for 2 hours at $37^{\circ} \mathrm{C}$ and $5 \%(\mathrm{v} / \mathrm{v}) \mathrm{CO} 2 \mathrm{in}$ air, before addition of $500 \mu \mathrm{l}$ culture medium. After 24 hours a further, $500 \mu \mathrm{l}$ of culture medium containing either the diluted chemical or culture medium alone was added.

End points

The resazurin reduction assay, kenacid blue assay and differentiation assay were performed.

\section{Cellular differentiation:}

The cultures were inspected morphologically for cardiomyocyte contractile activity at 24,48 and 144 hours under light microscope and observations were made according to the scoring method shown in table 1. Resazurin reduction assay

The resazurin assay was performed on day 6 following explantation. The medium was removed from the 24 well plates and replaced with 500 $\mu 1$ resazurin solution. The plates were then incubated for one hour at $37 \mathrm{oC}$ and $5 \%(\mathrm{v} / \mathrm{v}) \mathrm{CO} 2$ in air. The optical density was read using a FLUOR star plate reader, excitation wavelength of $-530 \pm 12.5 \mathrm{~nm}$, with a gain of 10 .

\section{Kenacid blue total protein assay}

The same cells subjected to the resazurin reduction assay were assayed for total protein using the kenacid blue assay. Wells were aspirated and $300 \mu \mathrm{KB}$ fixative was added and allowed to evaporate overnight at $4 \mathrm{oC}$. KB working solution $(400 \mu \mathrm{l})$ was added to each well and the plate placed on a plate shaker for at least 2 hours. Excess stain was removed and cells were quickly rinsed before being washed in $400 \mu$ lo washing solution for 20 minutes with agitation. The washing solution was replaced with $400 \mu l$ of desorb and gently agitated on the plate shaker for one hour. The optical density was read on an ASYS HITEC Expert 96 plate reader with a reference filter of $405 \mathrm{~nm}$, and a reading filter of $570 \mathrm{~nm}$.

Test chemical preparation for in-ovo culture

The test chemicals were dissolved in PBS to give a desired concentration (Nicotine $10 \mu \mathrm{M}$, folic acid $1 \mathrm{mM}$, and vitamin $\mathrm{C} 100 \mu \mathrm{M})$. All chemicals were prepared and applied to chick embryos in ovo within 1 hour of preparation.

\section{Inovo culture}

White leghorn fertilized chicken eggs were incubated at $370 \mathrm{C}$ in $100 \%$ humidity. At day 3 of incubation, eggs with viable embryos were either kept untouched or injected with or nicotine $(10 \mu \mathrm{M})$, vitamin $\mathrm{C}$ $(100 \mu \mathrm{M})$, folic acid $(1 \mathrm{mM})$, nicotine plus vitamin C or folic acid or PBS, by making a hole on their blunt end. Eggs were then sealed, labelled and re-incubated with the blunt end up till day 9 of incubation. On day 9 , 
embryos were taken out under sterile conditions and examined under a dissecting microscope for any gross malformations and scored according to the criteria shown in table described by Memon \& Pratten in 2009 [10].

\section{Histological staining}

Once examined grossly hearts from different groups were fixed in formalin $10 \%$ and prepared for histological sectioning as described previously [10].

Statistical analysis

Statistical analysis was performed using Prism 5 (Graph pad Software Inc. San Diego, USA). All results with different dose concentrations were compared using one way ANOVA with Dunnet's multiple comparison post hoc test, with $\mathrm{p}<0.05$ was considered statistically significant.

\section{Results of Nicotine treatment}

\section{Results}

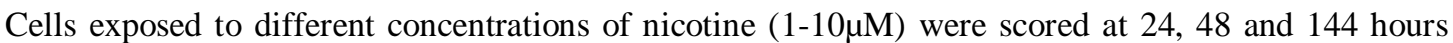
(Unpublished data). The graph shows that at $10 \mu \mathrm{M}$ nicotine, cardiomyocytes showed less contractile activity on day 2 and had no any beating at $122 \mathrm{hrs}$ post exposure (day 6). Statistical analyses showed that at 24 hours there was no significant difference between cell scores but this was to be expected as scoring was tested just before addition of chemicals. Statistical analyses of cell scores on day 2 and 6 showed a significant difference $(\mathrm{p}<$ $0.05)$ between cell scores at nicotine $(10 \mu \mathrm{M})$ and controls. Results also show that addition of folic acid $(1 \mathrm{mM})$ and vitamin $\mathrm{C}(100 \mu \mathrm{M})$ to nicotine treated cells can protect the cells from the embryotoxic effects of nicotine (fig 1 a).

The graph for the resazurin reduction assay shows a decrease in the amount of resorufin produced by nicotine treated cells $(10 \mu \mathrm{M})$, but that is not significantly different to controls (fig $1 \mathrm{~b}$ ). The results also show that the cells treated with addition of $1 \mathrm{mM}$ folic acid or $100 \mu \mathrm{M}$ vitamin $\mathrm{C}$ to nicotine are comparable to controls (fig $1 \mathrm{~b}$ ). The protein content of cardiomyocytes is also decreased at $10 \mu \mathrm{M}$ nicotine as shown in fig 1 (c). However statistical analyses showed no significant difference to controls. The results also show that the cells treated with folic acid and vitamins $\mathrm{C}$ in addition to nicotine were comparable to controls (fig. $1 \mathrm{c}$ ).

Table 1 Morphological scoring system to determine contractile activity of cardiomyocytes

\begin{tabular}{|l|l|}
\hline Numerical morphological score & Contractile activity \\
\hline 0 & No contractile activity \\
\hline 1 & Few contracting foci \\
\hline 2 & Numerous contracting foci \\
\hline 3 & Entire plate contracting \\
\hline
\end{tabular}

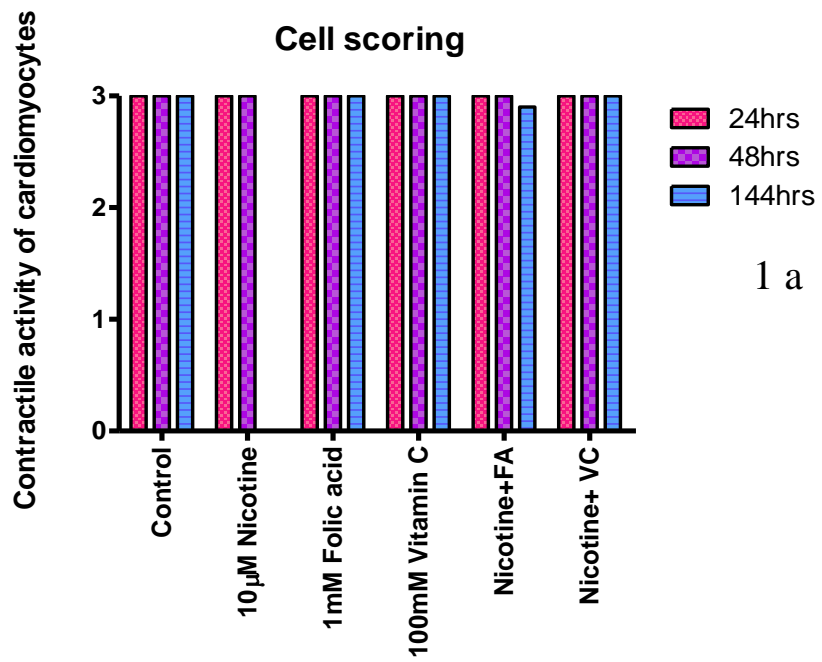



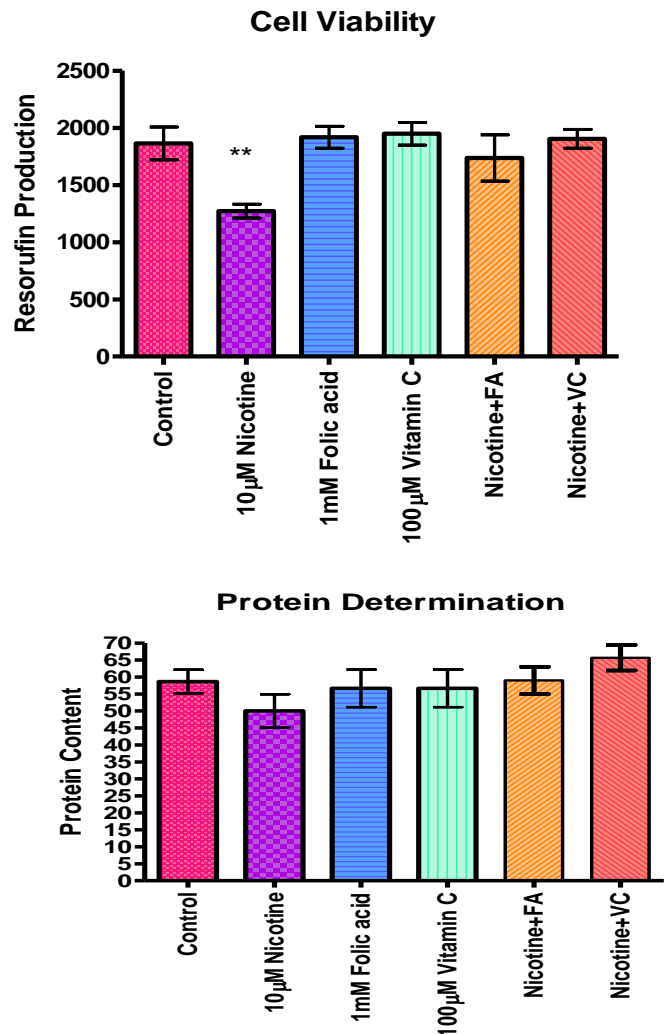

\section{Cadmium chloride with folic acid and vitamin $\mathrm{C}$}

Results show that addition of folic acid and vitamin $\mathrm{C}$ to cadmium chloride treated cells (5 and $10 \mu \mathrm{M})$ could not protect the cells from its embryotoxic effects. Fig 2a and 3a show contractile activity of cardiomyocytes. Cadmium chloride at both concentrations used decreased the beating and addition of folic acid and vitamin $\mathrm{C}$ has no protective effects on contractile activity which is decreased with cadmium chloride as shown in fig 2 a, 3a. Fig $2 \mathrm{~b}$ and $3 \mathrm{~b}$ show resorufin produced by cells. Results shows that addition of folic acid and vitamin $\mathrm{C}$ to cadmium chloride treated cells could not reverse the cytotoxic effects of cadmium chloride. Cellular protein levels, affected by cadmium chloride were also not rescued by addition of folic acid and vitamin $\mathrm{C}$ as shown in (fig $2 \mathrm{c}, 3 \mathrm{c}$ ).

Cell scoring
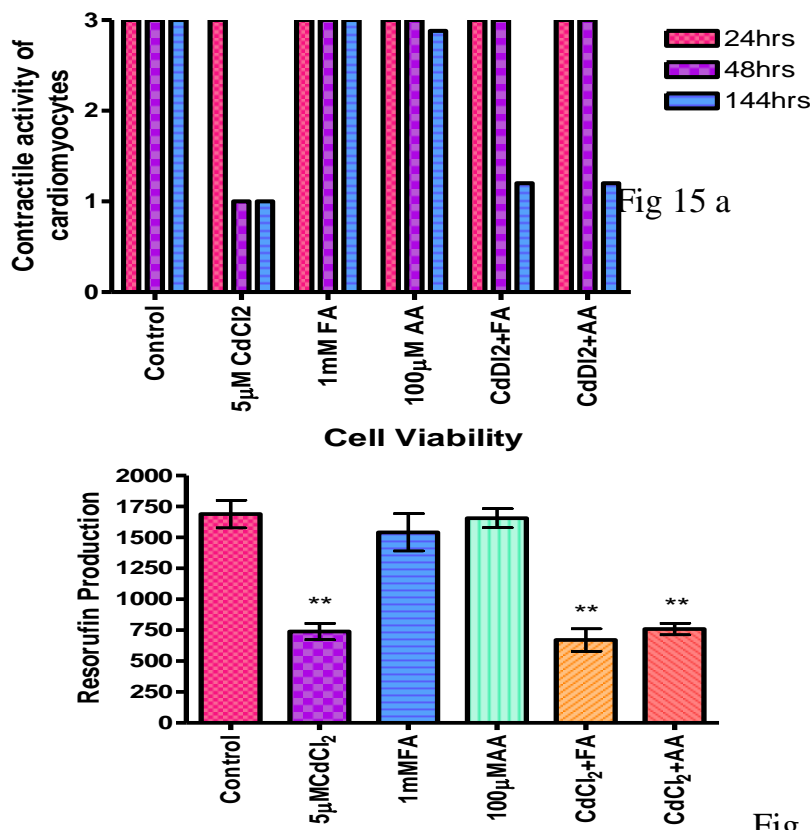

Fig $15 \mathrm{~b}$ 
Protein Determination

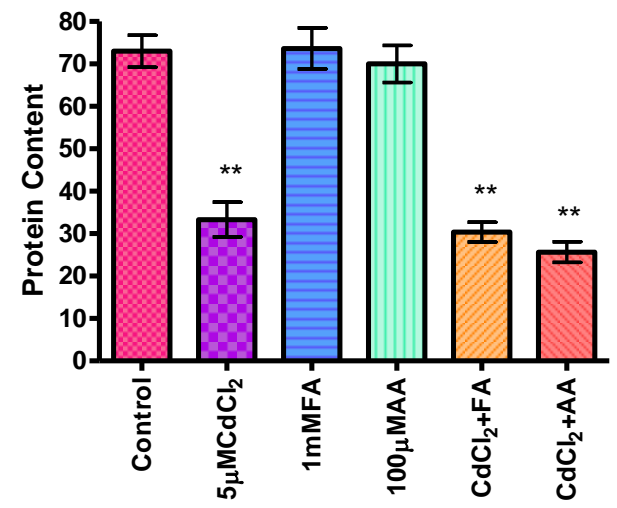

Fig 2. Shows contractile activity, cell viability and protein content of cardiomyocytes with $5 \mu \mathrm{M}$ of cadmium chloride plus folic acid and vitamin $\mathrm{C}(\mathrm{a}, \mathrm{b}, \mathrm{c})$.
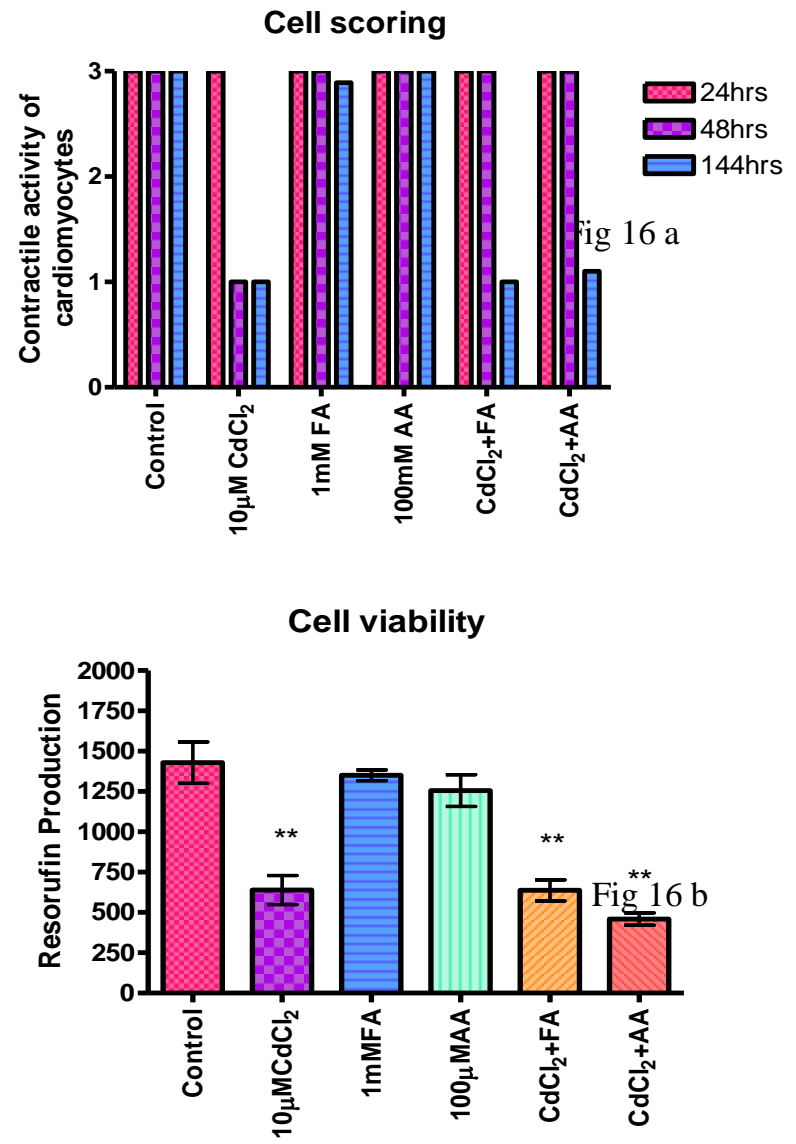


\section{Protein Determination}

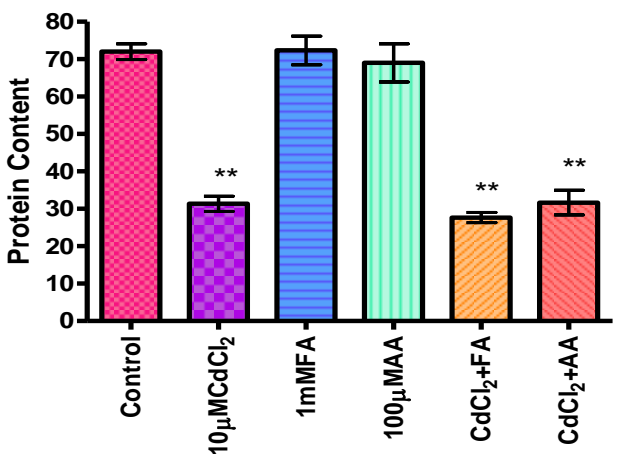

\section{Results of Nicotine treatment in Ovo culture}

The embryos treated with nicotine were growth retarded (fig $4 \mathrm{~b}$ ) as compared to embryos treated with PBS, which showed no gross abnormalities (Fig 4 a), However treatment of embryos with vitamin $\mathrm{C}$ and folic acid in addition to nicotine showed no gross malformations and none of them was growth retarded as shown in fig $4 \mathrm{c} \& \mathrm{~d}$ respectively. Histological examination of the hearts shows that hearts of nicotine treated embryos (fig $5 \mathrm{~b}$ ) show no abnormalities and were comparable to controls (fig 5 a). Also histological examination of hearts treated with additional vitamin $\mathrm{C}$ or folate were comparable to controls (fig $5 \mathrm{c} \& \mathrm{~d}$ respectively). Statistical analyses of the crown rump length of embryos showed a decrease in length of nicotine treated embryos ( $\mathrm{p}<0.05)$, while embryos with vitamin $\mathrm{C}$ and folic acid in addition grew normally and were comparable to control, as shown in fig 6. Moreover Statistical analyses of other parameters including vitelline circulation (fig 7 a), flexion of the embryo (fig 7 b), heart development (fig 7 c), and gross facial structures (fig 8 a), brain development (fig 8 b) and limb development (fig $8 \mathrm{c}$ ) show no significant difference between nicotine treated embryos, control and vitamin $\mathrm{C}$ and folic acid treated embryos.
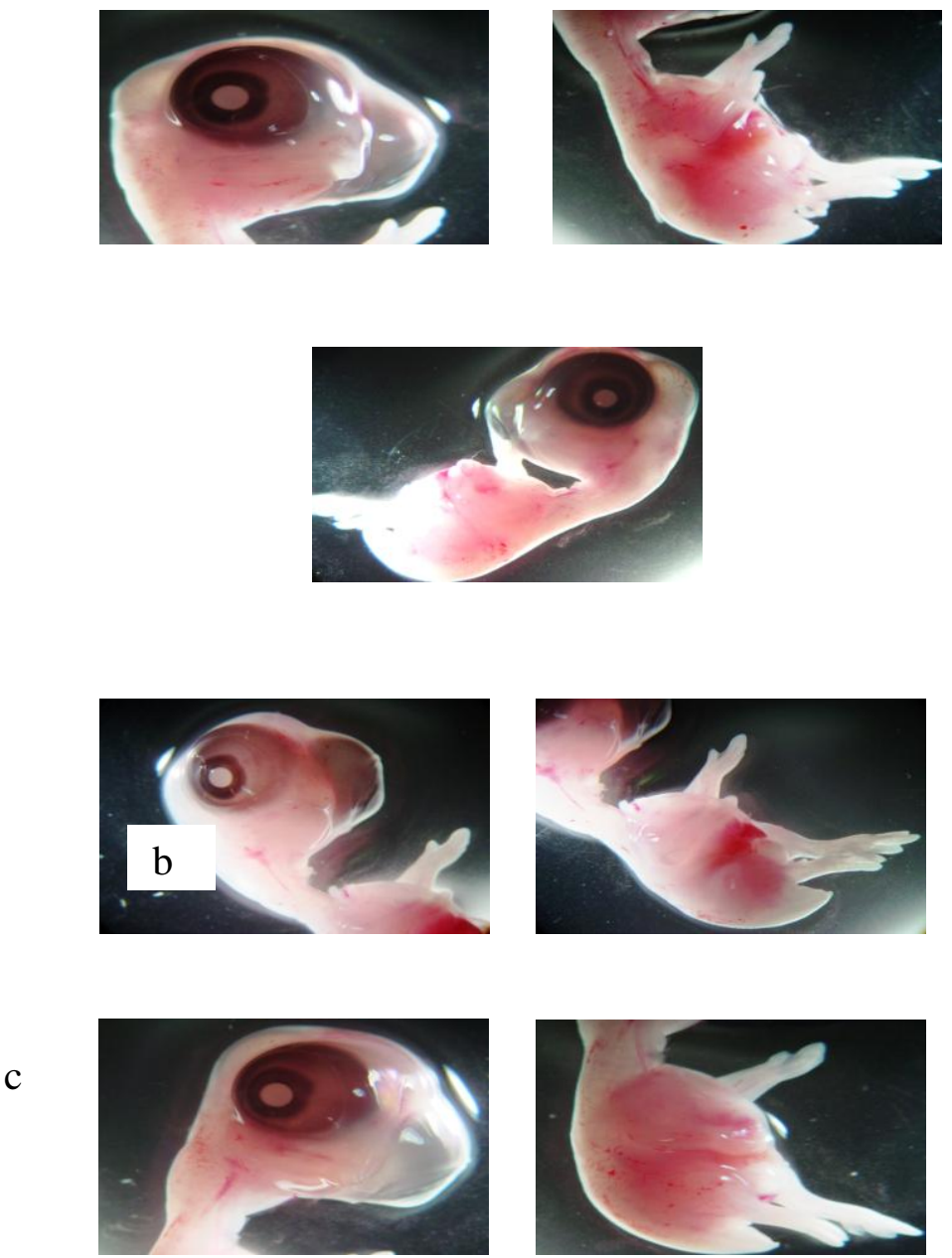
a

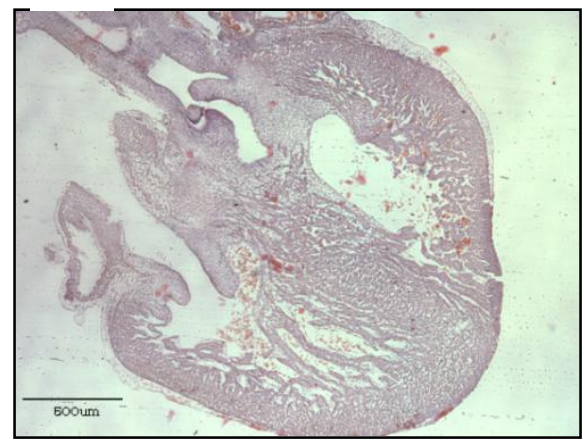

$\mathrm{c}$

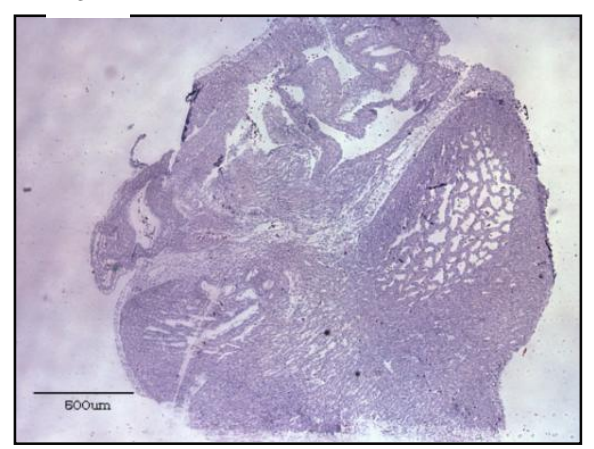

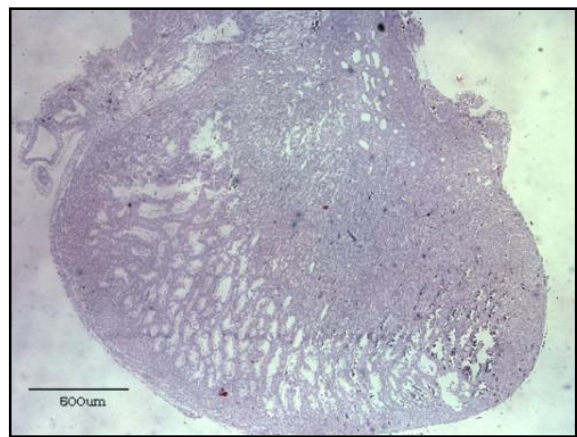

b

d

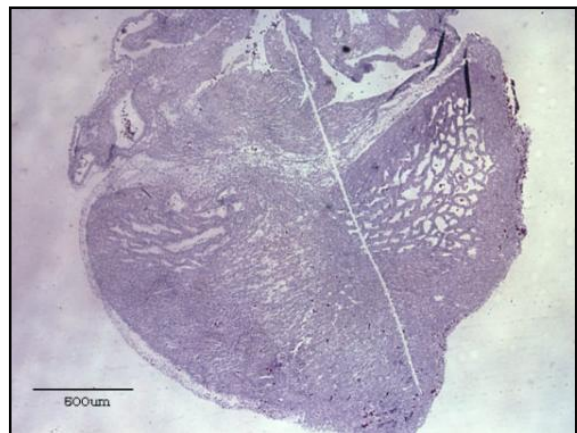

Figure 5: Shows H\&E staining of control hearts (a), treated with nicotine (b), nicotine plus vitamin $\mathrm{C}(\mathrm{c})$ and folic acid (d).

Crown Rump Length

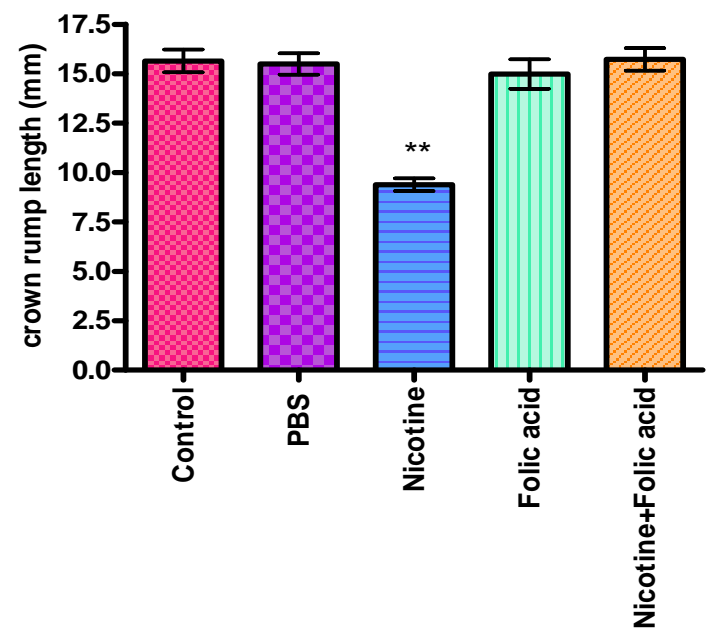

Crown Rump Length

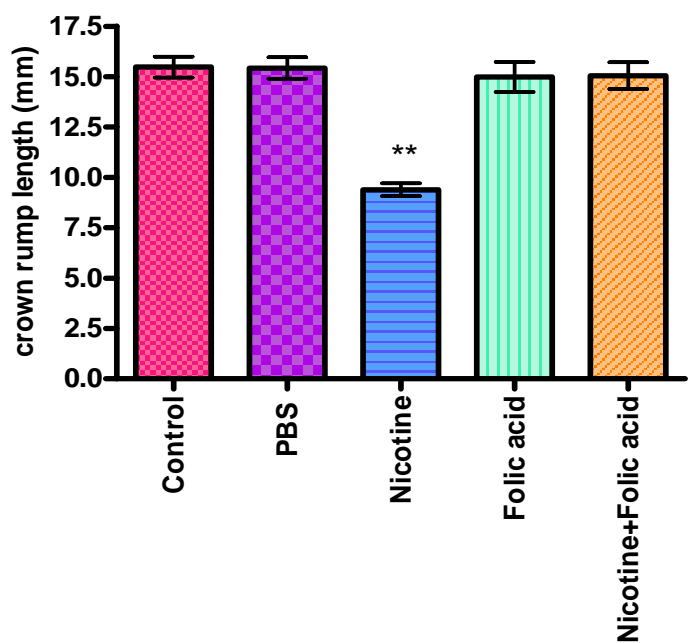

Figure 6: Shows crown rump of embryos treated with ethanol, plus vitamin $\mathrm{C}$ and folic acid. 
Vitteline Circulation

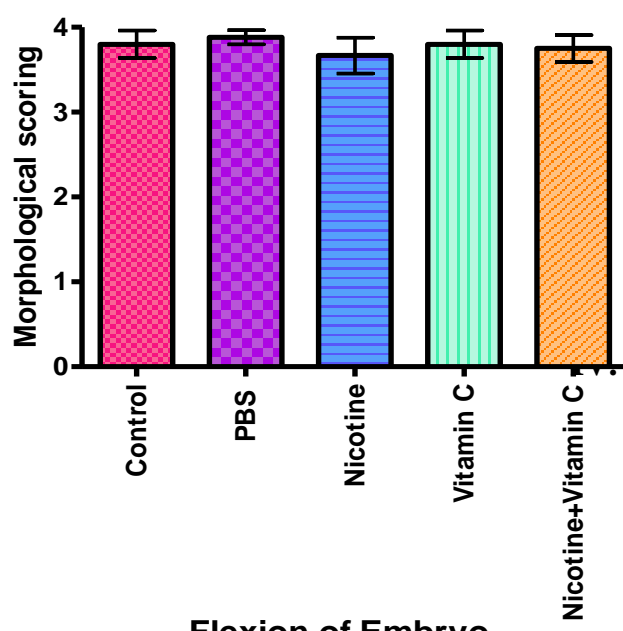

Flexion of Embryo

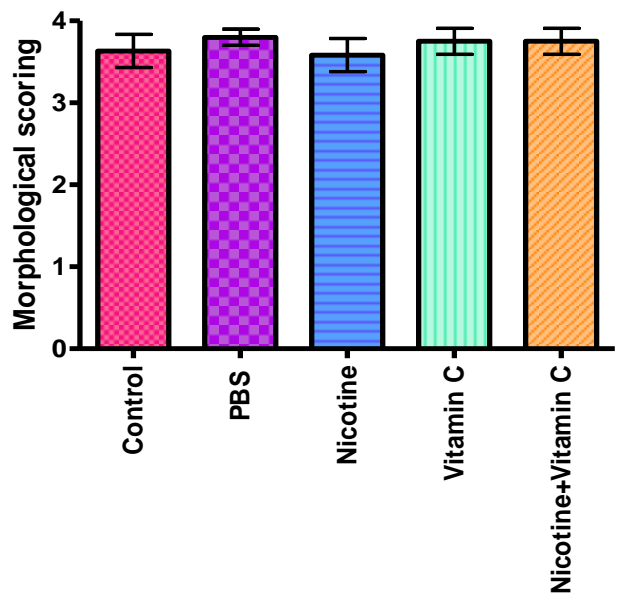

Heart Malformations

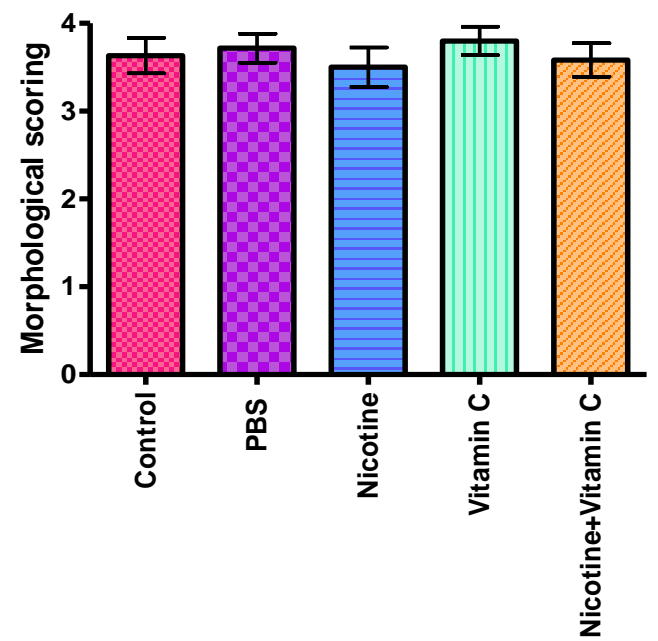

Vitteline Circulation

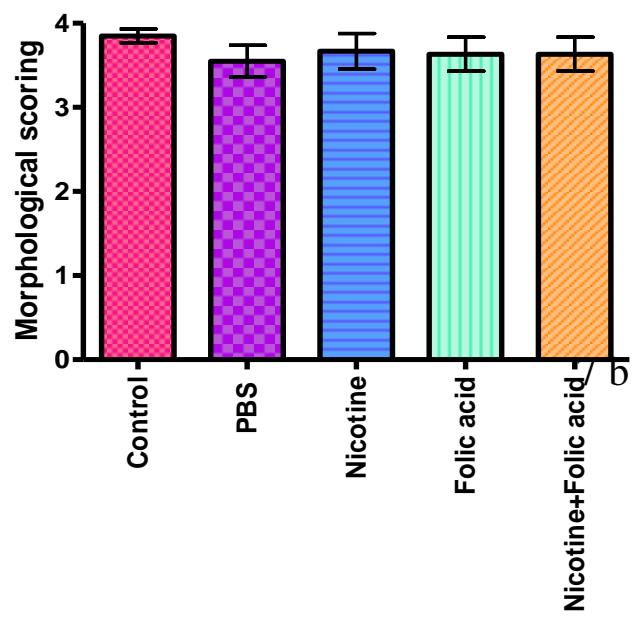

Flexion of Embryo
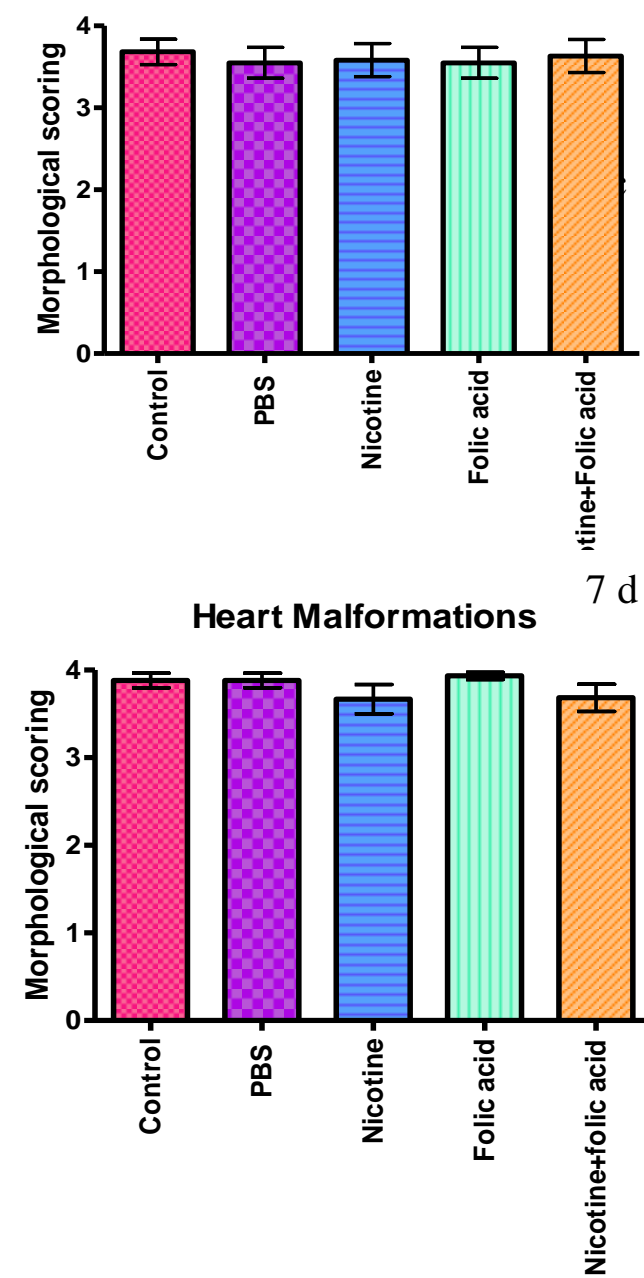

Figure 7: Shows vitalline circulation (a) flexion of embryo (b) and heart malformations (c) of embryos treated with nicotine, plus vitamin $\mathrm{C}$ and folic acid. 
8 a

Gross Fascial Malformations

Gross Fascial Malformation
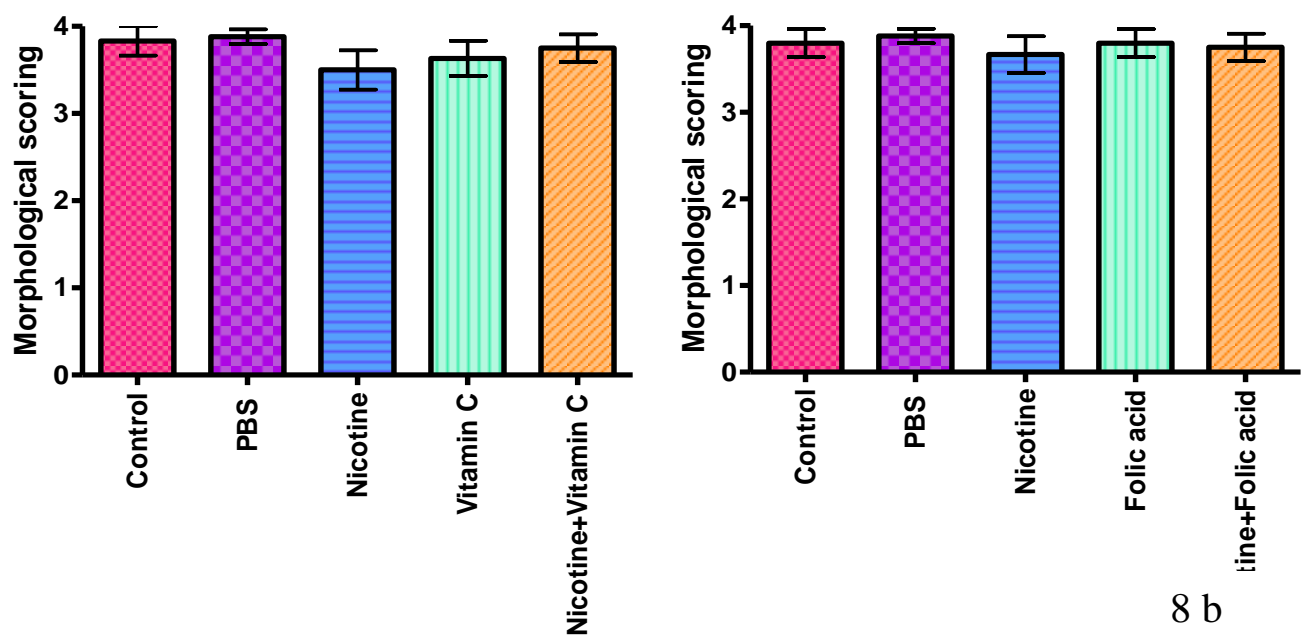

Brain Development
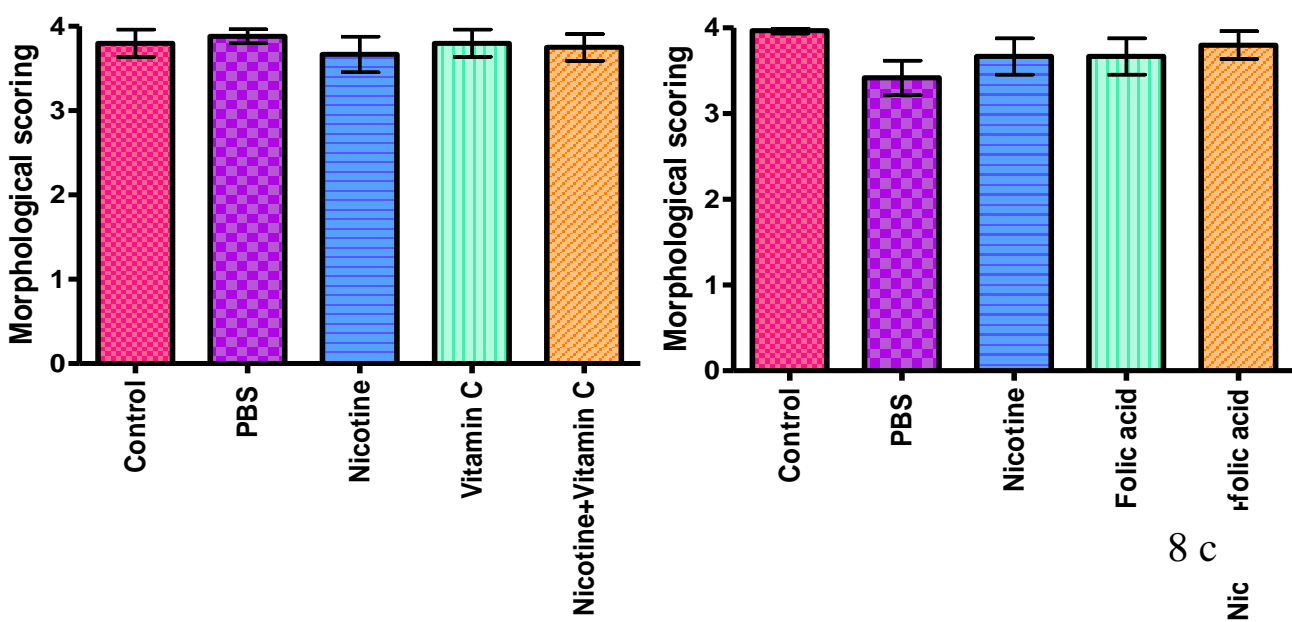

Limb Development

Limb Development
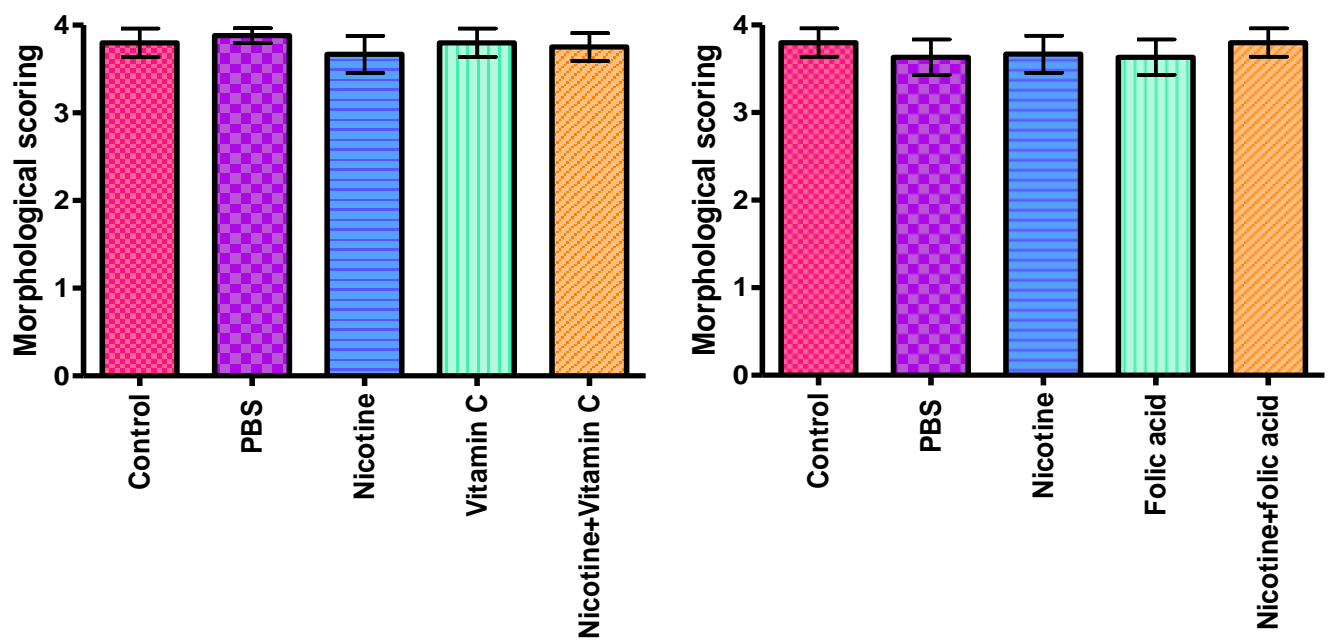

Figure 8: Shows facial malformations (a) brain development (b) and limb development (C) of embryos treated with nicotine, plus vitamin $\mathrm{C}$ and folic acid. 


\section{Discussion}

Avian embryos were chosen as a model system for this study as these are cost effective, easy to handle, less time consuming, involve no maternal sacrifice and provide reliable results. Cadmium chloride and nicotine are known human teratogens and therefore have been extensively investigated. This study was conducted to study cadmium chloride and nicotine induced developmental toxicity and also to investigate any protective effects of vitamin $\mathrm{C}$ and folic acid in preventing teratogenic effects brought about by ethanol and nicotine, comparing both micromass culture and in ovo culture using chick embryos. The teratogenic potential of cadmium on chick micromass culture, as well as in ovo culture, was confirmed by our present work. The effects were consistent for both culture systems used. While teratogenic effects of nicotine on chick micromass was confirmed in this study, the dose of nicotine which showed toxic effects on cardiomyocytes in culture had not shown toxic effects on chick embryos cultured in ovo, except growth retardation. Using the chick micromass system the effects observed were consistent for any of the three end points measured. The reliability of chick micromass culture was tested by Wiger et al [5] with many toxic chemicals used to treat the limb bud cells.

Nicotine administration has teratogenic effects on many animal species. In one study conducted by Zhao and Reece on mouse embryos, nicotine was shown to have teratogenic effects in a dose dependant manner. The mouse embryos treated with nicotine were growth retarded [26]. These findings are consistent with this study as the majority of embryos treated with nicotine were growth retarded. However in another study conducted on rat embryos show that carbon monoxide was the major contributing factor for low fetal birth weight [27]. The epidemiological studies conducted on humans also showed a relationship between tobacco smoking and congenital malformations. The results of one study conducted in the Baltimore-Washington area, show an association between maternal cigarette smoking of more than one pack per day and cardiovascular malformations, including: transposition of great vessels with ventricular septal defect and pulmonary stenosis. Similarly a relationship between maternal smoking and increased risk of neonates born with congenital heart diseases was observed in a case-control study conducted in Lithuania [228]. However some epidemiological studies also show no association between smoking and congenital malformations [29, $\underline{30}]$.

The present study also demonstrates a protective effect of Vitamin $\mathrm{C}$ and folic acid on cadmium and nicotine treated cardiomyocytes in vitro as well as in ovo. The effective concentration of vitamin $\mathrm{C}$ shown to have a protective effect is $100 \mu \mathrm{M}$ which is consistent with the amount of vitamin $\mathrm{C}$ used by Peng et al [31] to treat ethanol induced growth retardation and microcephaly in Xenopus laevis embryos. The concentration of folic acid with protective effect is $1 \mathrm{mM}$ which is similar to the amount of folinic acid used to treat the malformations of branchial arch derived structures in rats in whole embryo culture by Zhang et al by [32]. In a case control study, conducted in Atlanta a direct relationship of prevention of cardiac defects, especially outflow tract and ventricular septal defects, with periconceptional use of multivitamins containing folic acid and vitamin $\mathrm{C}$ was suggested [33-35]. Similar results were also found in a randomized control trial in Hungary which showed a reduction of 50\% in cardiac defects with supplemental folic acid during pregnancy [36].

The observations that supplemental vitamin $\mathrm{C}$ and folic acid offered a remarkable protection against the effects of nicotine might be due to their antioxidant properties, as has been shown by animal studies conducted on chick and rat embryos $[37,38]$. These studies proposed an involvement of oxidative stress produced by the generation of free oxygen radicals when embryos were exposed to ethanol, and suggest a critical role for reactive oxygen species in the teratogenic actions of ethanol. Under these conditions, reactive oxygen species may be continuously formed as a by product of metabolic reactions. Furthermore such reactive oxygen species have also been linked with embryonic heart defects [39] caused by a variety of environmental toxins and maternal diseases. However the extent of reactive oxygen species production and the subsequent likelihood of a developmentally toxic effect depend on the time and dose of exposure. Therefore, in order for normal development of embryos to be achieved under conditions where free radicals or reactive oxygen species are generated at abnormally high levels, there must be a balance between free radical generation and Vitamin $\mathrm{C}$, which may be useful in promoting degradation of free radicals [40]. In fact embryos have less protective mechanisms against free radicals compared to adults [41], therefore antioxidant supplements, like folic acid and Vitamin C, may be useful in protecting embryos against damage.

Furthermore results in this study suggest no preventive effects of folic acid and ascorbic acid against cadmium chloride induced developmental toxicity even at lower concentrations ( 5 and $10 \mu \mathrm{M})$. There are many mechanisms involved in cadmium induced teratogenecity; one possible mechanism could be the generation of reactive oxygen species as studies conducted on mouse and human promonocytic cells showed that prior treatment with $\alpha$-tocopherols and $\mathrm{N}$-acetyle-L-cysteine could prevent these cells from cadmium induced toxicity [42]. Another possible mechanism could be due to activation of calcium dependant endonucleases, as cadmium cation may mimic calcium channels and treatment with a calcium channel blocker could protect the cells from cadmium induced cell death [43]. 


\section{Conclusion:}

This study shows the toxic effects of nicotine and cadmium in micromass culture which showed efficiency of this system for prediction of teratogens. Also protective effects of multivitamins were observed in prevention of nicotine induced toxicity in both systems. While these antioxidants did not show any protection against cadmium toxicity in micromass culture, due to the possibility of other mechanisms involved in cadmium induced cardiovascular abnormalities.

\section{References}

[1]. Spielmann, H., et al., Preliminary results of the ECVAM validation study on three in vitro embryotoxicity tests. Altern. Lab. Anim, 2001. 29: p. 301-303.

[2]. Flint, O.P. and T.C. Orton, An invitro assay for teratogens with cultures of rat embryo midbrain and limb bud cells. Toxicology and Applied Pharmacology, 1984. 76: p. 383-395.

[3]. Parsons, J.F., J. Rockley, and M. Richold, In vitro micromass teratogen test: Interpretation of results from a blind trial of 25 compounds using three separate criteria. Toxicology in Vitro, 1990. 4(4-5): p. 609-611.

[4]. Tsuchiya, T., et al., Embryolethality of new herbicides is not detected by the micromass teratogen tests 1991. 65(2): p. 145-149.

[5]. Wiger, R., A. Strottum, and G. Brunborg, Estimating chemical developmental hazard in chicken embryo limb bud micromass system. Pharmacol Toxicol., 1988. 62(1): p. 32-37.

[6]. L'Huillier, N., M.K. Pratten, and R.H. Clothier, The relative embryotoxicity of 1,3-dichloro-2-propanol on primary chick embryonic cells. Toxicology in Vitro, 2002. 16(4): p. 433-442.

[7]. Meyer, M.P., et al., The Extracellular ATP Receptor, cP2Y1, Inhibits Cartilage Formation in Micromass Cultures of Chick Limb Mesenchyme. Developmental Dynamics, 2001. 222: p. 494-505.

[8]. Hurst, H.S., R.H. Clothier, and M. Pratten, An evaluation of a novel chick cardiomyocyte micromass culture assay with two teratogens/embryotoxins associated with heart defects. ATLA, 2007. 35(5): p. 505-511.

[9]. Ahir, B. and M.K. Pratten, Association of anxiolytic drugs diazepam and lorazepam, and the antiepileptic valproate, with heart defects--effects on cardiomyocytes in micromass (MM) and embryonic stem cell culture. Reprod Toxicol., 2011. 31(1): p. 66-74.

[10]. Memon, S. and M.K. Pratten, Developmental toxicity of ethanol in chick heart in ovo and in micromass culture can be prevented by addition of vitamin C and folic acid. . Reprod Toxicol, 2009. 28(2): p. 262-269.

[11]. Memon, S. and M.K. Pratten, Teratogenic effects of diabetic conditions in chick heart in ovo and micromass culture may be prevented by addition of vitamin C and folic acid. Reprod Toxicol, 2013. 35: p. 117-124.

[12]. Atterwill, C.K., H. Johnston, and S.M. Thomas, Models for the in vitro assessment of neurotoxicity in the nervous system in relation to xenobiotics and neurotrophic factor-mediated events. Neurotoxicology, 1992. 13: p. 39-53.

[13]. Vahter, M., et al., Integrated personal monitoring of cadmium exposure in Sweden. IARC Sci Publ, 1992. 118: p. 113-119.

[14]. Gilani, S.H. and Y. Alibhai, Teratogenicity of metals to chick embryos. Journal of Toxicology and Environmental Health, 1990. 30(1): p. $23-31$

[15]. Thompson, J., et al., Effects of cadmium on cell death and cell proliferation in chick embryos. Reproductive Toxicology, 2005. 20(4): p. 539-548.

[16]. Chen, B.Y. and B.F. Hales, Cadmium-Induced Rat Embryotoxicity in Vitro Is Associated with an Increased Abundance of ECadherin Protein in the Yolk-Sac. Toxicology and Applied Pharmacology, 1994. 128(2): p. 293 -301.

[17]. Jeong, S.-H., S.S.M. Habeebu, and C.D. Klaassen, Cadmium Decreases Gap Junctional Intercellular Communication in Mouse Liver. Toxicol. Sci., 2000. 57(1): p. 156-166.

[18]. Sunderman, F.J., M.C. Plowman, and S.M. Hopfer, Teratogenicity of cadmium chloride in the South African frog, Xenopus laevis. IARC Sci Publ., 1992. 118: p. 249-256.

[19]. Hen Chow, E.S. and S.H. Cheng, Cadmium Affects Muscle Type Development and Axon Growth in Zebrafish Embryonic Somitogenesis. Toxicol. Sci., 2003. 73(1): p. 149-159.

[20]. Chan, P.K. and S.H. Cheng, Cadmium-induced ectopic apoptosis in zebrafish embryos Arch Toxicol, 2003. 77: p. 69-79.

[21]. Jauniaux, E. and G.J. Burton, Morphological and biological effects of maternal exposure to tobacco smoke on the feto-placental unit. Early Human Development, 2007. 83(11): p. 699-706.

[22]. Morales-Suarez Varela, M.M., et al., Socio-occupational status and congenital anomalies. Eur J Public Health, 2009. 19(2): p. 161 167.

[23]. Ermak, G. and K.J.A. Davies, Calcium and oxidative stress: from cell signaling to cell death. Molecular Immunology, 2002. 38(10): p. 713-721.

[24]. Gumustekin, K., et al., Vitamin E but not Hippophea rhamnoides L. prevented nicotineinduced oxidative stress in rat brain. Hum. Exp. Toxicol, 2003. 8: p. 425-431.

[25]. Kelly, G., The interaction of cigarette smoking and antioxidants. Part III. Ascorbic acid. Altern. Med. Rev., 2003. 8(1): p. 43-54.

[26]. Zhao, Z. and E.A. Reece, Nicotine-Induced Embryonic Malformations Mediated by Apoptosis From Increasing Intracellular Calcium and Oxidative Stress. Birth Defects Research (Part B), 2005. 74: p. 383-391.

[27]. Carmines, E.L. and N. Rajendran, Evidence for Carbon Monoxide as the Major Factor Contributing to Lower Fetal Weights in Rats Exposed to Cigarette Smoke. Toxicol. Sci., 2008. 102(2): p. 383-391.

[28]. Kučienè, R. and V. Dulskienè, Selected environmental risk factors and congenital heart defects. Medicina (Kaunas), 2008. 44(11): p. 827-831.

[29]. Scherbak Y., et al., Smoking influence on birth of child with birth defects in big city (Kyiv, Ukraine), in In: Jedrychowski WA, Perera FP, Maugeri U.Vulnerability of fetus and infant to ambient pollutants and reduced food intake in pregnancy2007, Jagiellonian University Press: Krakow. p. 242.

[30]. Kallen, K., Maternal smoking and congenital heart defects. European Journal of Epidemiology : , 1999. 15: p. $731-737$.

[31]. Peng, Y., et al., Ascorbic acid inhibits ROS production, NF-[kappa]B activation and prevents ethanol-induced growth retardation and microencephaly. Neuropharmacology, 2005. 48(3): p. 426-434.

[32]. Zhang, Z., et al., Prevention of retinoic acid-induced early craniofacial abnormalities by folinic acid and expression of endothelin1/dHAND in the branchial arches in mouse. British journal of nutrition, 2006. 96: p. 418-425.

[33]. Botto, L.D., J. Mulinare, and J.D. Erickson, Do mutivitamin or folic acid supplements reduce the risk for congenital heart defects? American Journal of Medical Genetics, 2003. 121A: p. 95-101.

[34]. Willcox, B.J., J.D. Curb, and B.L. Rodriguez, Antioxidants in Cardiovascular Health and Disease: Key Lessons from Epidemiologic Studies. The American Journal of Cardiology, 2008. 101(10, Supplement 1): p. S75-S86. 
[35]. Botto, L.D., R.S. Olney, and J.D. Erickson, Vitamin supplements and the risk for congenital anomalies other than neural tube defects. Am J Med Genet C Semin Med Genet, 2004. 125: p. 12-21.

[36]. Gardinar, H.M., Keeping abreast of advances in fetal cardiology. Early human development, 2006. 82: p. 415-419.

[37]. Henderson, G.I., et al., In Utero Ethanol Exposure Elicits Oxidative Stress in the Rat Fetus. Alcoholism: Clinical and Experimental Research, 1995. 19(3): p. 714-720.

[38]. Nasha, C.M., et al., Effects of maternal administration of vitamins $\mathrm{C}$ and $\mathrm{E}$ on ethanol neurobehavioral teratogenicity in the guinea pig. Alcohol, 2007. 41: p. 577-586.

[39]. Hobbs, C.A., et al., Congenital heart defects and maternal biomarkers of oxidative stress. American Journal of Clinical Nutrition, 2005. 82(3): p. 598-604

[40]. Barrow, H.M., M.J. Jean, and P. Michael, Amelioration of Ethanol-Induced Neurotoxicity in the Neonatal Rat Central Nervous System by Antioxidant Therapy. Alcohol Effects on the Fetus, Brain, Liver, and Other Organ Systems. Alcoholism: Clinical \& Experimental Research. , 2000. 24(4): p. 512-518.

[41]. Reimers, M.J., et al., Ethanol-dependent toxicity in zebrafish is partially attenuated by antioxidants. Neurotoxicology and Teratology, 2006. 28(4): p. 497-508.

[42]. Galán, A., et al., Modulation of the stress response during apoptosis and necrosis induction in cadmium-treated U-937 human promonocytic cells. Biochimica et Biophysica Acta (BBA) - Molecular Cell Research, 2001. 1538(1): p. 38-46.

[43]. Azzouzi, B.E., et al., Cadmium induced apoptosis in a human T cell line. Toxicology, 1994. 88(1-3): p. 127-139. 